\title{
Near-Field Antenna Measurements Using Photonic Sensor of Mach-Zehnder Interferometer
}

\author{
Masanobu Hirose, Satoru Kurokawa, Michitaka Ameya, and Koji Komiyama
}

National Institute of Advanced Industrial Science and Technology, Tsukuba, Ibaraki 305-8568, Japan

Correspondence should be addressed to Masanobu Hirose, masa-hirose@aist.go.jp

Received 15 October 2011; Accepted 19 January 2012

Academic Editor: Amedeo Capozzoli

Copyright (๑) 2012 Masanobu Hirose et al. This is an open access article distributed under the Creative Commons Attribution License, which permits unrestricted use, distribution, and reproduction in any medium, provided the original work is properly cited.

\begin{abstract}
We have been developing a photonic sensor system to measure the electric near-field distribution at a distance shorter than one wavelength from the aperture of an antenna. The photonic sensor is a type of Mach-Zehnder interferometer and consists of an array antenna of $2.4 \mathrm{~mm}$ height and $2 \mathrm{~mm}$ width on $\mathrm{LiNbO}_{3}$ substrate $(0.5 \mathrm{~mm}$ thickness, $8 \mathrm{~mm}$ length, and $3 \mathrm{~mm}$ width $)$ supported by a glass pipe. The photonic sensor can be considered to be a receiving infinitesimal dipole antenna that is a tiny metallic part printed on a small dielectric plate at microwave frequency. Those physical and electrical features make the photonic sensor attractive when used as a probe for near-field antenna measurements. We have demonstrated that the system can be applied to planar, spherical, and cylindrical near-field antenna measurements without any probe compensation approximately below $10 \mathrm{GHz}$. We show the theories and the measurements using the photonic sensor in the three near-field antenna measurement methods.
\end{abstract}

\section{Introduction}

The techniques of near-field antenna measurements are the important and prevailing tools for accurate far-field pattern measurements for any kind of antennas [1]. Recently, in the EMC society, the pattern of an antenna used in the SVSWR method [2] is required from $1 \mathrm{GHz}$ up to $6 \mathrm{GHz}$ and it is known that the pattern has significant effects on the validation of test site above $1 \mathrm{GHz}$ and emission measurements [3]. Therefore a compact and easy-to-use equipment for a near-field antenna measurement is desired. The one of the candidates is the near-field antenna measurement system using a photonic sensor [4-6].

A few kinds of electrooptic probes are proposed to detect electric fields or magnetic fields in the air, near antennas or printed boards, and so on. A kind of the probes based on Mach-Zehnder interferometer uses some kinds of antennas on the $\mathrm{LiNbO}_{3}$ (LN) [7-9]. Another kind of the probes based on polarization modulation uses the crystal bulk [10-13]. Among the probes, we have used the photonic sensor shown below as the one of most promising probes from viewpoints of stability and cost.
The photonic sensor, which detects electric fields by the antenna, consists of a tiny dipole antenna on a $\mathrm{LiNbO}_{3}$ substrate whose operating principle is a Mach-Zehnder interferometer. The most important characteristic of the sensor is that the sensor can be treated as an infinitesimal dipole antenna working in receiving mode. The merits of the photonic sensor applied to near-field antenna measurements are

(1) no probe compensation is required below $10 \mathrm{GHz}$,

(2) broad band measurements from a few tens $\mathrm{MHz}$ to $10 \mathrm{GHz}$ are realized by the single probe,

(3) the sensor can be treated as the more ideal infinitesimal receiving antenna if the operating frequency becomes the lower,

(4) the truncation error can be reduced,

(5) evanescent waves in the immediate vicinity of the aperture can be detected,

(6) measurement system can be made in small and compact size. 


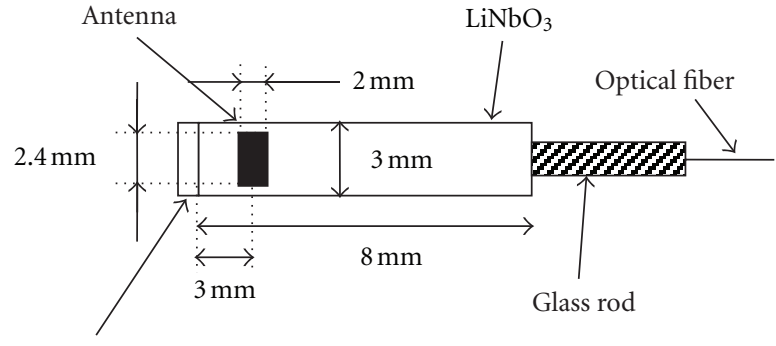

Mirror

(a)

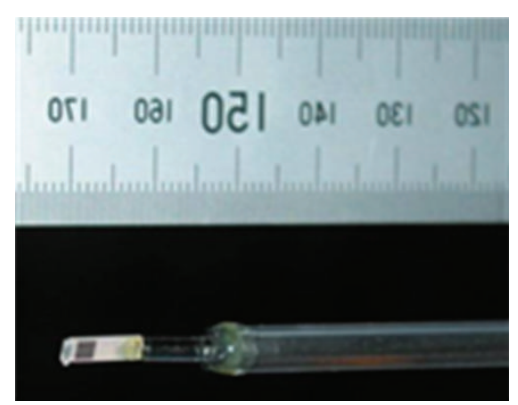

(b)

FIGURE 1: Structure of the latest photonic sensor: (a) size and (b) picture.

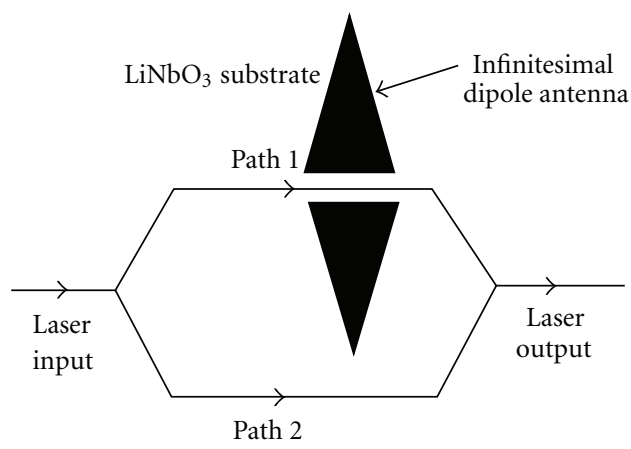

Figure 2: Principle of operation.

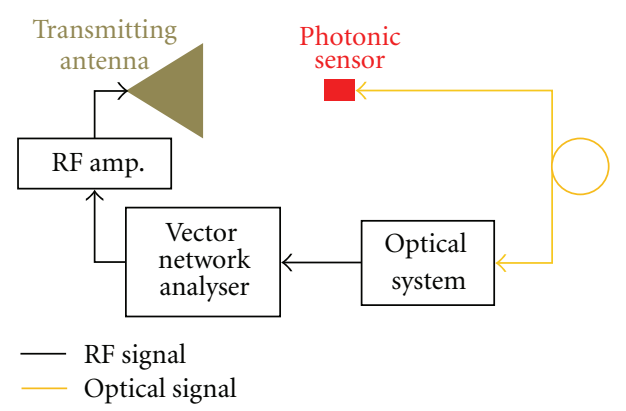

FIgURE 3: Schematic setup for measurements.
Section 2 shows the characteristics of the photonic sensor. Section 3 shows the formulations using the photonic sensor to planar, spherical, and cylindrical near-field antenna measurements and the measurement results for various kinds of antennas are compared with those by various methods. Finally the merits using the photonic sensor are summarized.

\section{Photonic Sensor}

2.1. Structure and Operating Principle. Our latest photonic sensor is shown in Figure 1 [5, 6]. A small metallic antenna on an LN substrate detects incident electric fields.

The antenna consists of an array of seven short metallic strip dipoles ( $2.4 \mathrm{~mm}$ high, $0.2 \mathrm{~mm}$ wide, $0.012 \mathrm{~mm}$ gap, and $0.1 \mathrm{~mm}$ apart from each element) printed on the LN substrate $(0.5 \mathrm{~mm}$ thick, $8 \mathrm{~mm}$ length, $3 \mathrm{~mm}$ width, $\mathrm{X}$-cut, and effective relative permittivity 28 ) that is connected to a glass rod (called ferrule) to join an optical fiber. The whole of the photonic sensor is supported by a glass pipe that is $5 \mathrm{~mm}$ in diameter and $1 \mathrm{~mm}$ thick. At the top of the photonic sensor, there is a block of a dielectric optical mirror that reflects laser beams on optical waveguides in the LN substrate.

Figure 2 explains the operating principle of the photonic sense whose structure is a type of Mach-Zehnder interferometer. The antenna in Figure 2 is a tiny (or infinitesimal) rhombic dipole antenna that was used as an old type of the photonic sensor. Of course, the principle is entirely the same as that in the latest photonic sensor.

A laser beam along an optical waveguide enters from the left side and splits into two beams at an optical Y-junction. The two beams are combined into one beam at the right-side of an optical Y-junction. Then the output laser beam is travelling along an optical fiber and detected by a photo-detector to convert an optical signal to a RF signal.

An external electric field excites a voltage at the centre gap of the dipole antenna. When the voltage is applied to one of the optical waveguides (the path 1 in Figure 2), the refractive index of the part of the LN substrate changes depending on the applied voltage due to the Pockels effect. It makes the optical length of the path 1 different from that of the path 2 . Since two beams through each path interfere with each other, the amplitude of the combined beam changes depending on the applied voltage. By detecting instantaneously the amplitude of the laser using the photodiode, we can measure the external electric field instantaneously.

The type of the photonic sensor that we use is called a reflection type because the dielectric mirror is placed between the antenna and the right-side of the Y-junction. Owing to this structure, the sensitivity of the sensor is doubled because the laser beam in the path 1 is modulated two times on a lap. 


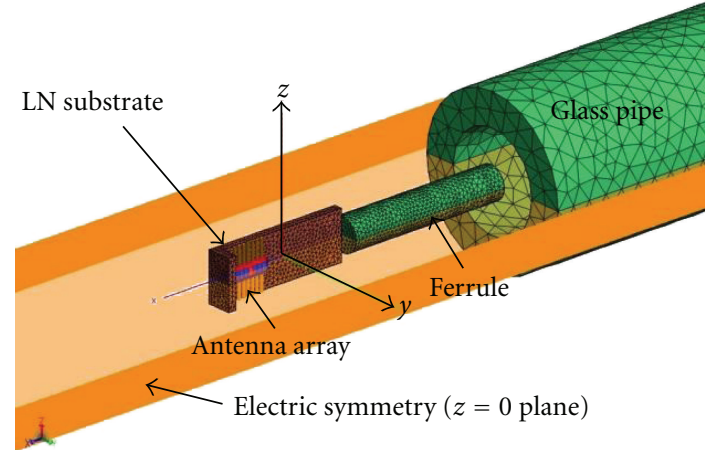

FIgUre 4: Model meshed by FEKO.

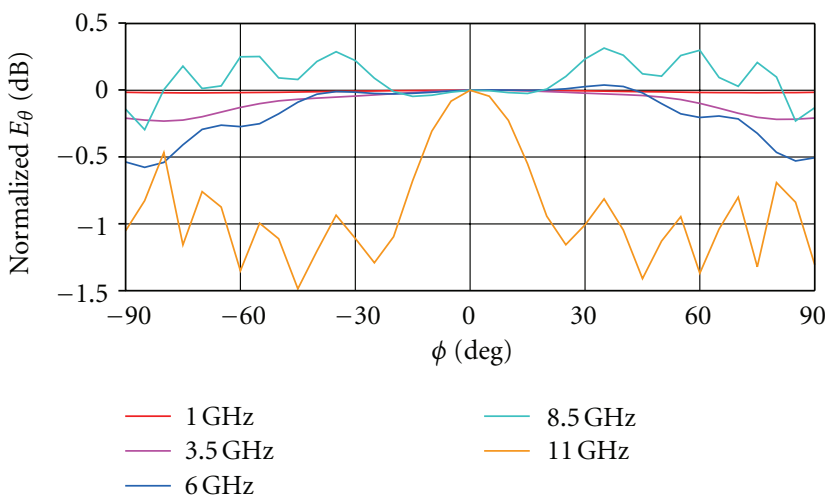

FIGURE 5: Normalized $E_{\theta}$ pattern in the $x-y$ plane.

2.2. Relation between Incident Electric Field and Measured $S_{21}$. Figure 3 shows the schematic setup for antenna measurements using the photonic sensor. The photonic sensor can detect the electric field radiated by the transmitting antenna at a small region or effectively a point of space where the sensor is placed because the antenna on the photonic sensor is infinitesimal effectively.

Therefore the $S_{21}$ between the transmitting antenna and the photonic sensor is measured by a vector network analyser (VNA) and expressed as

$$
S_{21}=\alpha \hat{\mathbf{p}} \cdot \frac{\mathbf{E}(\mathbf{r})}{a_{0}}
$$

where $\alpha$ is a constant including the error terms of the system and the refection coefficient of the transmitting antenna, $\hat{\mathbf{p}}$ is the unit vector parallel to the dipole moment vector of the photonic sensor, $\mathbf{E}(\mathbf{r})$ is the electric field at a position vector $\mathbf{r}$, and $a_{0}$ is the complex amplitude of the incoming wave into the antenna.

From the plane-wave scattering-matrix theory of antennas [14], we have proved (1) clearly in [4]. More intuitively, the same equation can be derived from the concept of the open voltage of the antenna.

If the antenna on the photonic sensor is at transmitting mode, the current $\mathbf{J}_{p}$ on the antenna is considered to be concentrated at a point effectively because the antenna is

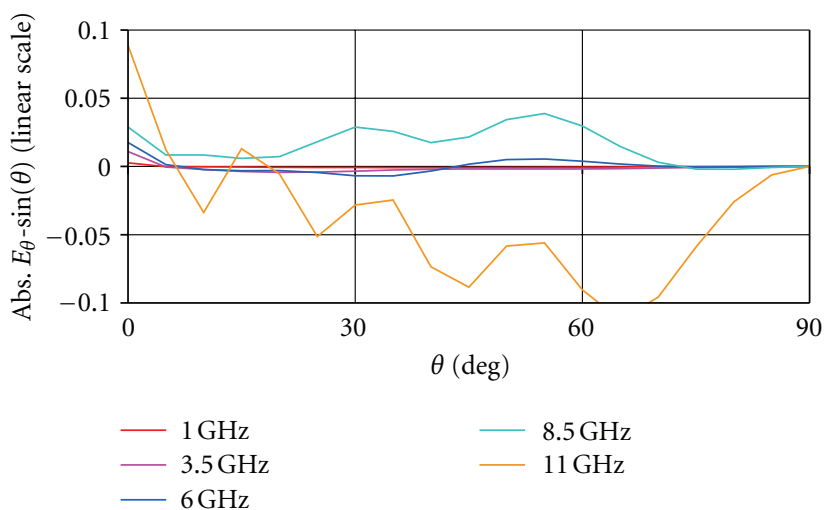

FIGURE 6: Difference between normalized $E_{\theta}$ and $\sin \theta$ in the $z-x$ plane. Linear scale.

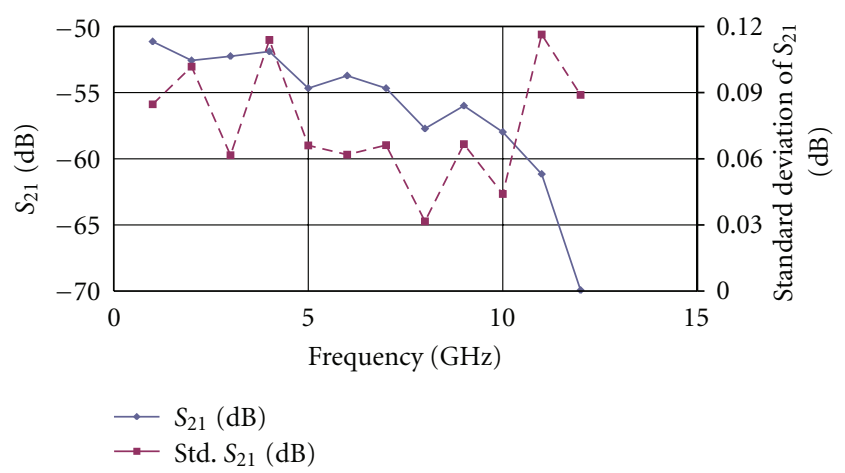

FIGURE 7: Frequency characteristics and the standard deviation of $S_{21}$ of the present antenna measurement system using the photonic sensor.

effectively infinitesimal. On the assumption, the open voltage $V_{\text {open }}$ induced on the antenna is approximated as [15]

$$
\begin{aligned}
-V_{\text {open }} I_{\text {in }} & =\int \mathbf{E}\left(\mathbf{r}+\mathbf{r}^{\prime}\right) \cdot \mathbf{J}_{p}\left(\mathbf{r}^{\prime}\right) e^{j \mathbf{k} \cdot \mathbf{r}^{\prime}} d \mathbf{r}^{\prime} \\
& \cong \mathbf{E}(\mathbf{r}) \cdot \int \mathbf{J}_{p}\left(\mathbf{r}^{\prime}\right) e^{j \mathbf{k} \cdot \mathbf{r}^{\prime}} d \mathbf{r}^{\prime} \\
& =\mathbf{E}(\mathbf{r}) \cdot(\alpha \hat{\mathbf{p}}),
\end{aligned}
$$

where $I_{\text {in }}$ is the input current of the antenna and $\mathbf{r}^{\prime}$ is the position vector whose origin is the centre of the antenna. Therefore we obtain the same equation as (1) intuitively.

From the above discussion, it is important to determine the maximum frequency where the photonic sensor is treated as an infinitesimal antenna. For the purpose, we have calculated the antenna pattern using the electromagnetic simulator (FEKO [16]).

Figure 4 shows the model after meshing and the electric symmetry at $z=0$ plane. The relative dielectric constants of the LN substrate, the ferrule, and the glass pipe are assumed to be 28,4 , and 4 , respectively. The corresponding mesh sizes are $0.3 \mathrm{~mm}, 0.3 \mathrm{~mm}$, and $1.2 \mathrm{~mm}$ respectively. The excited antenna is the centre in the array of seven elements. 


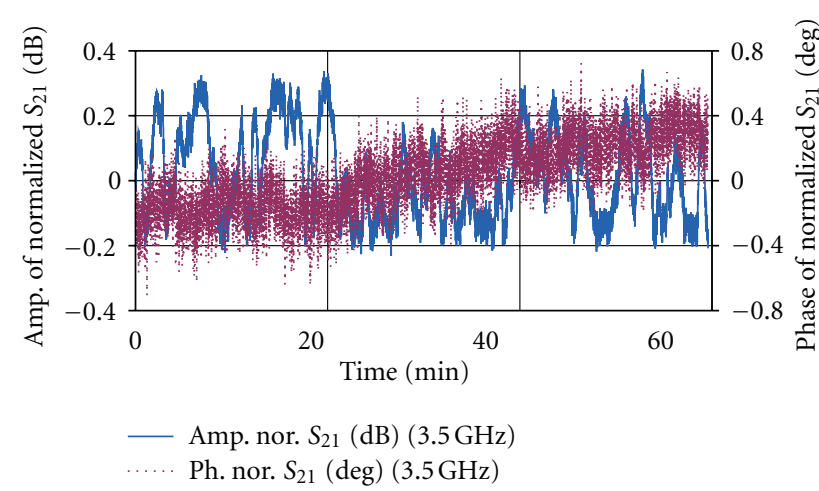

FIgURE 8: Long-term stability of the amplitude and phase of $S_{21}$ normalized by the average value at $3.5 \mathrm{GHz}$ (worst case).

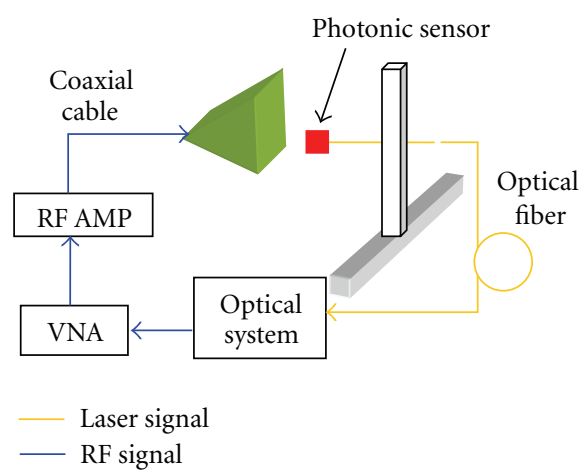

FIgURE 9: Measurement setup using planar scanning.

Figure 5 shows the normalized $E_{\theta}$ pattern in the $x-y$ plane. Figure 6 shows the difference between the normalized $E_{\theta}$ and $\sin \theta$ in the $z-x$ plane. If the antenna is an infinitesimal antenna, the patterns in the $x-y$ plane and the $z-x$ plane should be isotropic and $\sin \theta$, respectively. Therefore the normalized $E_{\theta}$ pattern should be $0 \mathrm{~dB}$ in Figure 5 and the difference should be zero in Figure 6 if the antenna is infinitesimal. From Figures 5 and 6, we can consider the photonic sensor as infinitesimal below $8 \mathrm{GHz}$ approximately. The deviation from the infinitesimal dipole is mainly due to the glass pipe to support the sensor. Then if the glass pipe is replaced to a styrene foam, the maximum frequency (for the old sensor) is over $10 \mathrm{GHz}$ from the calculation by FEKO.

2.3. Sensitivity and Stability of System. The sensitivity of the photonic sensor is so low that an RF amplifier must be used in antenna measurements because the power of a conventional VNA is at most $10 \mathrm{dBm}$. The minimum sensitivity of the sensor is approximately $0.5 \mathrm{~V} / \mathrm{m}$ at $2.45 \mathrm{GHz}$. Therefore, to obtain the SNR of $S_{21}$ larger than $40 \mathrm{~dB}$ at peak level, the input power greater than $30 \mathrm{dBm}$ or $1 \mathrm{~W}$ is required for a standard horn antenna whose gain is $17 \mathrm{dBi}$ at $2.45 \mathrm{GHz}$.

The frequency characteristics of the system including every characteristics of the antenna (ETS-Lindgren 3115), the coaxial cables (about $10 \mathrm{~m}$ ), the optical fiber $(50 \mathrm{~m})$, and the RF amplifier are shown in Figure 7. The standard deviation is calculated in the time interval of 3 minutes. The

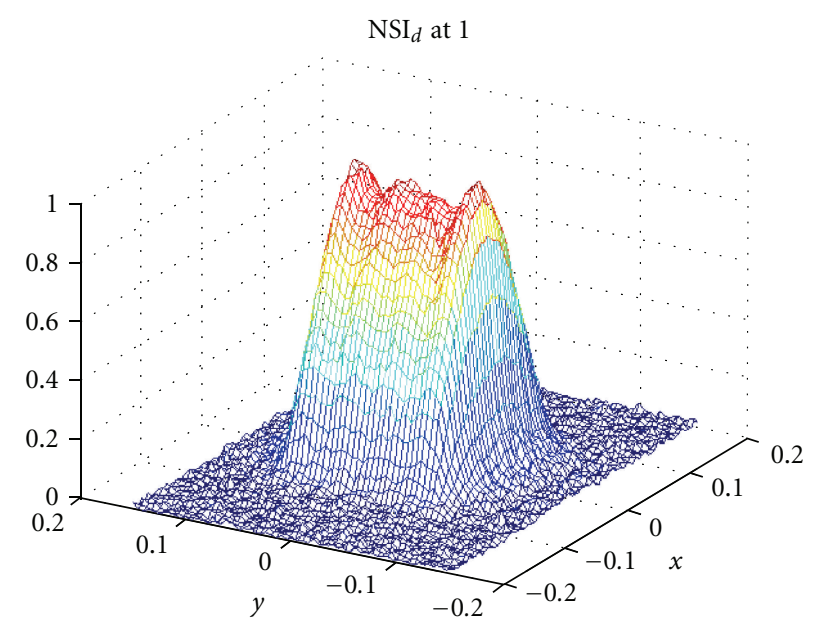

Figure 10: The amplitude of $S_{21}$ at $10 \mathrm{~mm}$ apart from the aperture of the standard horn antenna measured by the old type of the photonic sensor.

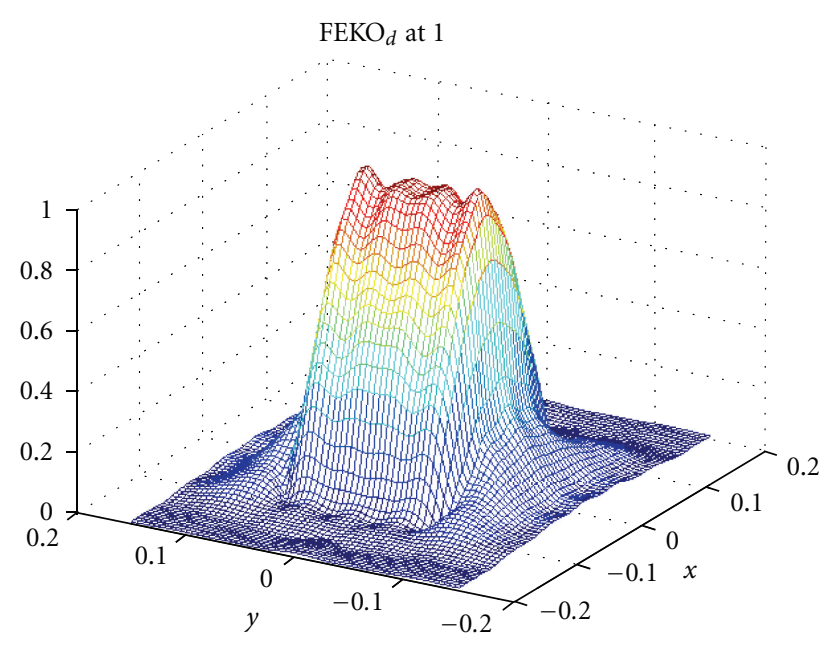

Figure 11: The amplitude of $E_{y}$ at $10 \mathrm{~mm}$ apart from the aperture of the standard horn antenna calculated by FEKO.

conditions of each component of the system are described below: the gain of the RF amplifier (HP8348A) is about $35 \mathrm{~dB}$, the cable loss is less than $5 \mathrm{~dB}$ at $2.45 \mathrm{GHz}$, the output power and the IF of the VNA (Agilent E8363C) are set to be $0 \mathrm{dBm}$ and $100 \mathrm{~Hz}$, respectively, the distance between the aperture of the antenna and the photonic sensor is $63 \mathrm{~mm}$. Since the sensor is set in right front of the centre of the antenna aperture, the measured level is almost at maximum.

The measured $S_{21}$ becomes worse above $11 \mathrm{GHz}$ because of the characteristic of the inner RF amplifier of the optical controller (Seiko Giken OEFS-S1). We have verified that the photonic sensor itself can be used up to $18 \mathrm{GHz}$ using another optical system.

The long-term (1 hour) stability at $3.5 \mathrm{GHz}$ (worst case) is shown in Figure 8 because the measuring time of a few hours is a typical in the near-field measurement using the photonic sensor. The standard deviation of the amplitude 


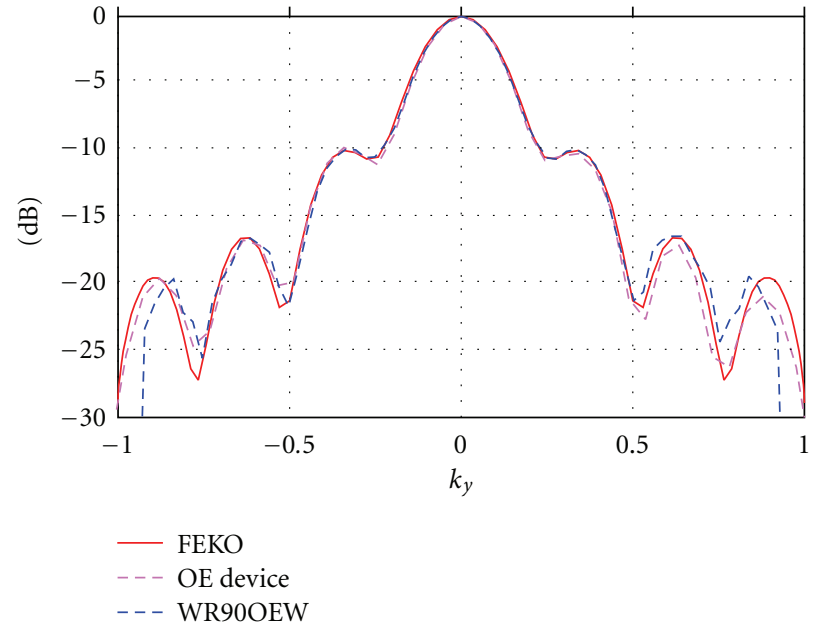

(a) E-plane pattern

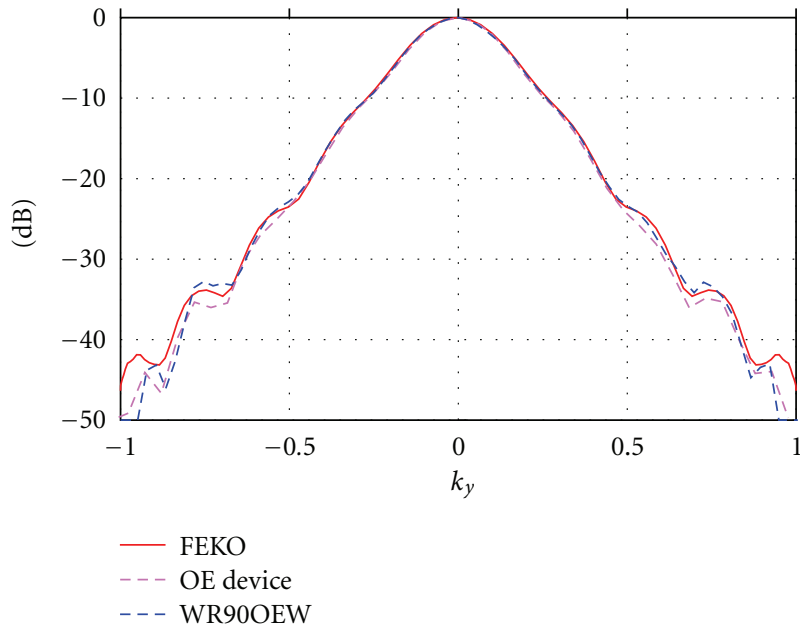

(b) H-plane pattern

FIGURE 12: Normalized patterns of the standard horn antenna obtained by the old photonic sensor (OE device), the WR90 OEW probe, and FEKO. (a) E-plane pattern. (b) H-plane pattern.

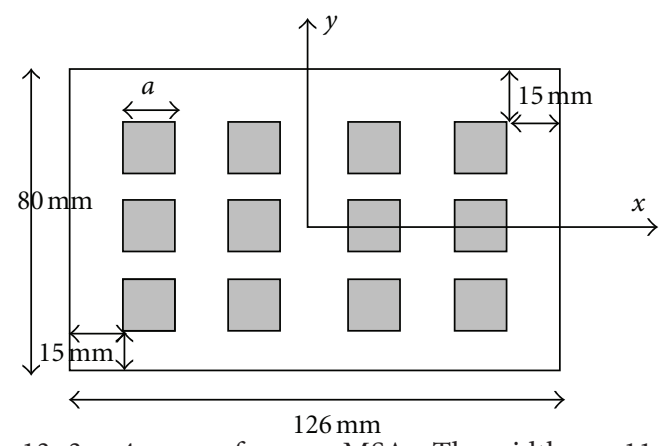

Figure 13: $3 \times 4$ array of square MSAs. The width $a=11 \mathrm{~mm}$ for each antenna.

and phase of $S_{21}$ is $0.14 \mathrm{~dB}$ and 0.23 degrees. In Figure 8 , the amplitude changes about $0.4 \mathrm{~dB}$ in a few minutes.

Those abrupt changes probably are due to the stability of the photonic sensor and the optical controller. However, the amplitude is constant on average. The phase on average increases linearly about 0.4 degrees in 1 hour. Because the instability can be considered as a kind of noise, the effect of the instability on the far-field pattern can be calculated by $[17,18]$ for planar near-field measurements and [19] for cylindrical near-field measurements.

After all, the photonic sensor can be used as an infinitesimal antenna up to approximately $8 \mathrm{GHz}$ and measure an incident electric field over $0.5 \mathrm{~V} / \mathrm{m}$ at $0 \mathrm{~dB}$ of $\mathrm{SNR}$ deduced from Figure 8.

To increase the sensitivity about ten times, we have a plan to insert an optical amplifier with low noise figure between the sensor and the photodiode to increase the modulated laser beam.

\section{Near-Field Measurements}

Using the photonic sensor system, we have measured various kinds of antennas in planar, spherical, and cylindrical scanning techniques successfully. In all scanning techniques, we assume the photonic sensor to be infinitesimal. Therefore no probe compensations are used in the formulations of the scanning techniques.

3.1. Planar Near-Field Measurements. A standard horn antenna (Mode 12-8.2 manufactured by Scientific Atlanta). The aperture size is $194 \mathrm{~mm}$ ( $\mathrm{H}$ plane $) \times 144 \mathrm{~mm}$ ( $\mathrm{H}$ plane). The antenna was measured using the planar scanner (Model 200 V-3x3 manufactured by NSI) as shown in Figure 9.

The photonic sensor used was an old type and the antenna shape was the same as that in Figure 2. However the sensitivity is almost the same as the present one because the base size of the rhombic antenna $(1.5 \mathrm{~mm})$ is comparable to the present size $(1.4 \mathrm{~mm}=0.2 \mathrm{~mm} \times 7)$ and the old sensor was also a reflection type [20].

Figure 10 shows the amplitude of the measured $S_{21}$ at the $10 \mathrm{~mm}$ plane apart from the aperture using the old type of the photonic sensor. In the following, the distance between the photonic sensor and the antenna aperture is defined as that between the centre of the array or the antenna on the sensor and the aperture. The amplitude is normalized relative the maximum. The antenna polarization of the sensor is along in the $y$-axis. The frequency is $8 \mathrm{GHz}$ and the scan area is $\pm 200 \mathrm{~mm}$ (the $x$ axis or $\mathrm{H}$ plane) $x \pm 150 \mathrm{~mm}$ (the $y$-axis or E plane) with $5 \mathrm{~mm}$ steps.

Figure 11 shows $E_{y}$ component of the electric field at $10 \mathrm{~mm}$ apart from the aperture calculated by FEKO. The meshing size is approximately $1 / 9$ wavelengths. The amplitude is normalized relative to the maximum and the scan area is the same as the one of the measurement in Figure 10.

Comparing Figures 10 and 11, we can conclude that the photonic sensor can be considered to be infinitesimal at $8 \mathrm{GHz}$. This is compatible with the conclusion deduced from Figures 5 and 6 . 


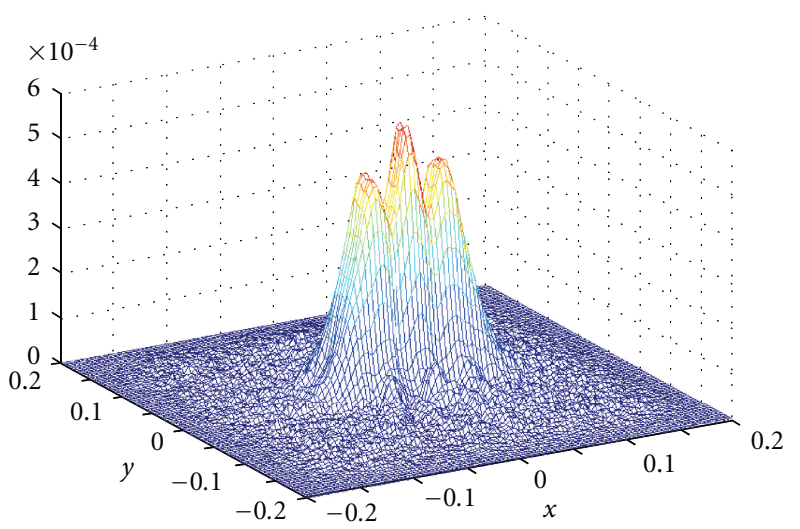

(a) $E_{x}$

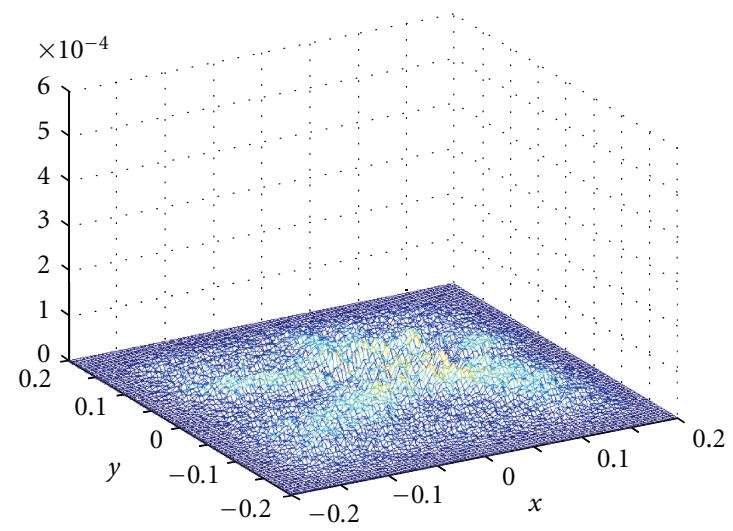

(b) $E_{y}$

FIGURE 14: The amplitudes of the measured $S_{21}$ corresponding to (a) $E_{x}$ and (b) $E_{y}$ at $10 \mathrm{~mm}$ apart from the antenna aperture using the old photonic sensor.

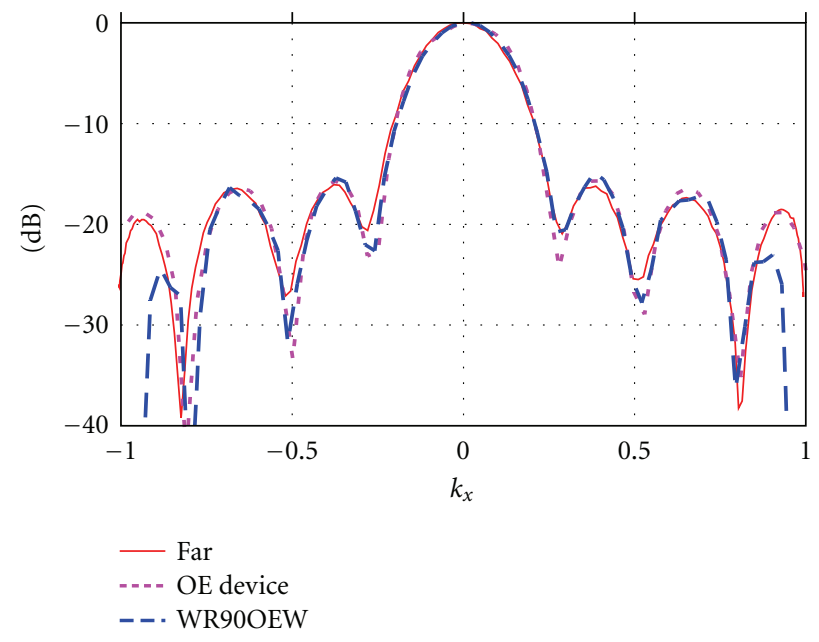

(a) E-plane

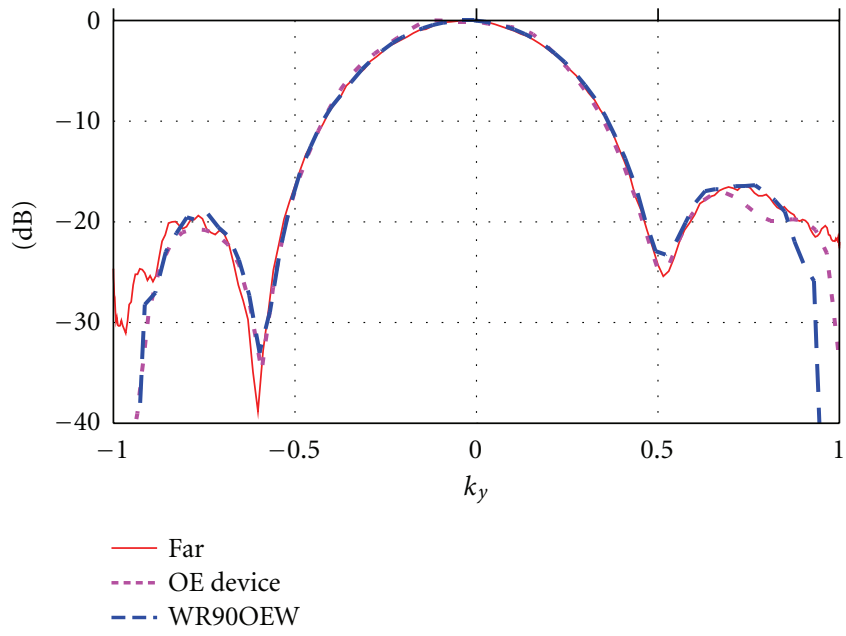

(b) H-plane

FIGURE 15: Normalized patterns of the array of the MSAs obtained by the old photonic sensor (OE device), the OEW probe (WR90OEW), and far-field measurement (Far). (a) E-plane pattern. (b) H-plane pattern.

From (1), an antenna pattern $D(\mathbf{K})$ is simply obtained by the Fourier transform of the measured $S_{21} s$ corresponding to the $E_{x}$ and $E_{y}$ components as

$$
\begin{gathered}
S_{21 x} \mathbf{x}+S_{21 y} \mathbf{y}=\iint \mathbf{F}(\mathbf{K}) e^{-j \mathbf{k} \cdot \mathbf{r}} d \mathbf{K}, \\
D(\mathbf{K})=\alpha_{p}|\mathbf{k} \times \mathbf{F}(\mathbf{K})|^{2},
\end{gathered}
$$

where $S_{21 x}, S_{21 y}$ are the measured $S_{21}$ s corresponding to the $E_{x}$ and the $E_{y}$ respectively. $\alpha_{p}$ is a constant, $\mathbf{x}$ and $\mathbf{y}$ are unit vectors for each directions, $\mathbf{k}$ is the wavenumber vector, and $\mathrm{K}$ is its projection vector on the $x-y$ plane.

The E-plane and the H-plane patterns obtained by the near-field to far-field transformations without probe compensation are shown in Figure 12. For the references, the patterns calculated by FEKO and the patterns obtained by the conventional open-ended waveguide probe (OEW) are also shown in the same figure. The near-field data measured by the OEW was measured at $110 \mathrm{~mm}$ apart from the aperture in the scan area of $\pm 326 \mathrm{~mm} \times \pm 326 \mathrm{~mm}$ ( $18 \mathrm{~mm}$ steps). The probe compensation was done using the pattern of the OEW (WR 90). The patterns by the three methods agree well each other.

As another type of antenna, we have measured an array $(3 \times 4)$ of microstrip antennas (MSA) shown in Figure 13 $[4,21]$. Each square MSA is coupled electromagnetically through thin slotted apertures to the feeding microstrip lines at the back of the antenna. The polarization of the array is along the $x$ axis.

Figure 14 shows the amplitudes of the measured $S_{21}$ for the $E_{x}$ and the $E_{y}$ components of the array at $10 \mathrm{~mm}$ apart from the antenna plane. The frequency is $9.41 \mathrm{GHz}$. That 


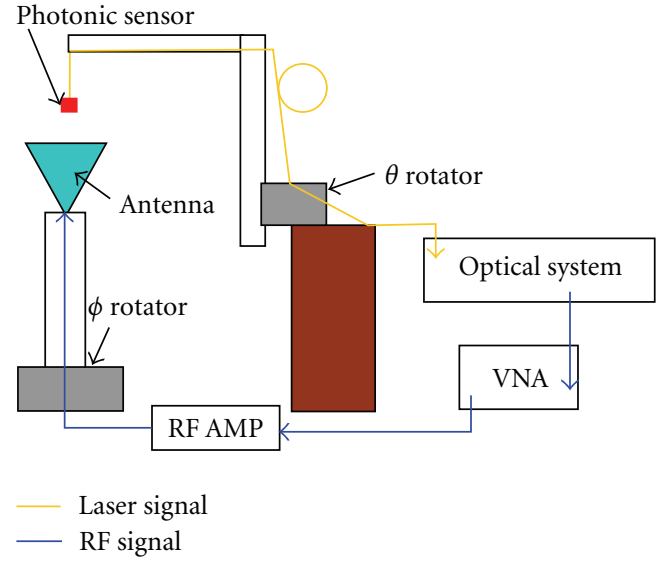

FIGURE 16: Measurement setup for spherical near-field scanning.

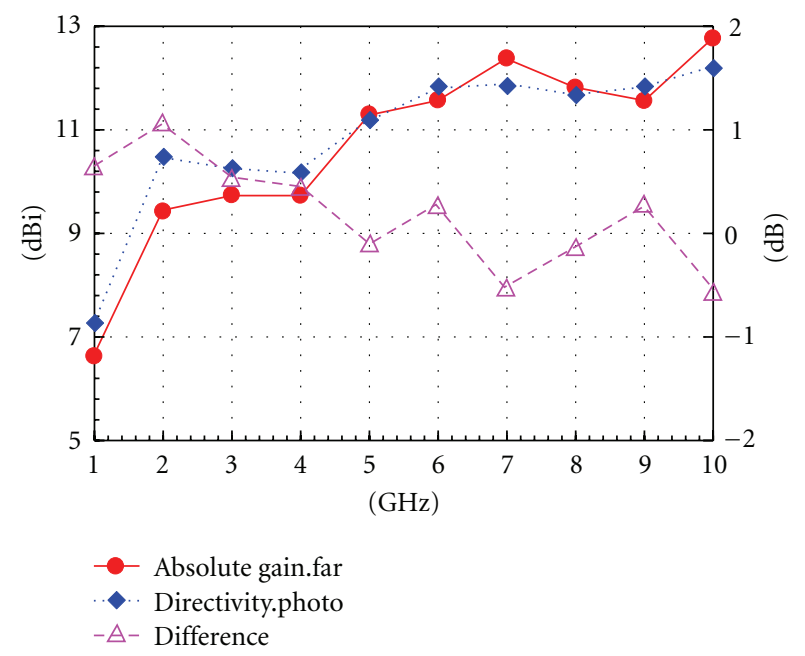

Figure 17: Absolute boresight gains (dBi) obtained by the far-field three antenna method (far) and the proposed method using the photonic sensor (photo).

means that the polarizations of the photonic sensor are along the $x$ axis and $y$ axis respectively. The scanned area is $\pm 200 \mathrm{~mm} \times \pm 200 \mathrm{~mm}$ and the sampling interval is $4 \mathrm{~mm}$.

The peaks of $E_{x}$ is at the centres of the spaces of the antennas along the $x$-axis. This is because the radiation by the MSA comes from the radiation edge of the antenna. This is different from the cosine tapered electric-field distribution of the horn antenna in Figure 10.

Figure 15 compares the patterns measured by the photonic sensor (OE device), the conventional far-field method (Far), and the WR90 OEW probe. The OEW measurement done in the scan is of $67.2 \mathrm{~cm} \times 67.2 \mathrm{~cm}$, at the distance of $110 \mathrm{~mm}$ between the aperture of the OEW and that of the antenna plan. We found that the patterns by the three measurement methods agree well with each other.

From the results, we conclude that the old photonic sensor can be considered to be an infinitesimal receiving antenna below approximately $10 \mathrm{GHz}$. This is not contradicting with Figures 5 and 6 because the photonic sensor (old) used in the above measurements was supported by a styrene foam block instead of the glass pipe that is used for the latest photonic sensor. The effect of the styrene foam on the pattern of the old photonic was calculated by FEKO and proved to be negligible even at $10 \mathrm{GHz}$.

3.2. Spherical Near-Field Measurements. Absolute gain measurements using the photonic sensor are easily realized in the spherical near-field measurements [22]. The measurements require an antenna with unknown gain, unknown pattern, however almost $100 \%$ efficiency. It is easy to realize an antenna with almost $100 \%$ efficiency if the antenna is composed by all metallic parts such as a standard horn antenna [23]. Since the efficiency of $98 \%$ equals to loss less than $0.1 \mathrm{~dB}$, the assumption of at almost $100 \%$ means the error less than $0.1 \mathrm{~dB}$, which is acceptable in many measurements.

Measurement setup is shown in Figure 16. The system was designed by ourselves. All parts irradiated by the antenna under measurement are covered by electromagnetic absorbers. The polarization of the photonic sensor can be adjusted to the theta or phi direction of the measurement coordinate system.

From (1), the measured $S_{21}$ s corresponding to the $E_{\theta}$ and $E_{\phi}$ components are represented as

$$
\begin{aligned}
& S_{21 \theta}=\alpha^{\prime} \sum_{s=1}^{2} \sum_{n=1}^{N} \sum_{m=-n}^{n} T_{s m n} \vec{F}_{s m n}^{(3)}(R, \theta, \phi) \cdot \hat{\theta}, \\
& S_{21 \phi}=\alpha^{\prime} \sum_{s=1}^{2} \sum_{n=1}^{N} \sum_{m=-n}^{n} T_{s m n} \vec{F}_{s m n}^{(3)}(R, \theta, \phi) \cdot \hat{\phi},
\end{aligned}
$$

where $\hat{\theta}, \hat{\phi}$ are unit vectors. $\alpha^{\prime}$ is equal to $\alpha k \sqrt{Z_{\text {free }}}$, where $Z_{\text {free }}$ is the free space impedance. $T_{\text {smn }}$ is the coefficient for the spherical wave function, $\vec{F}_{s m n}^{(3)}(R, \theta, \phi)[24]$, and $R$ is the distance between the photonic sensor and the centre of the measurement coordinate system. For our system, $R$ is typically $60 \mathrm{~cm}$ approximately.

$\alpha^{\prime} T_{s m n}$ is determined by the numerical integration as

$$
\begin{aligned}
\alpha^{\prime} T_{s m n}= & (-1)^{m}\left\{R_{s n}^{(3)}(k R)\right\}^{-2} \\
& \times \int_{\phi=0}^{2 \pi} \int_{\theta=0}^{\pi}\left\{S_{21 \theta} \hat{\theta}+S_{21 \phi} \hat{\phi}\right\} \cdot \vec{F}_{s,-m, n}^{(3)}(R, \theta, \phi) \\
& \times \sin \theta d \theta d \phi,
\end{aligned}
$$

where $R_{s n}^{(3)}(k R)$ is the radial function [24].

On the other hand, the gain of the antenna is given as

$$
G(\theta, \phi)=\frac{\left|\sum_{s m n} \alpha^{\prime} T_{s m n} \vec{K}_{s m n}(\theta, \phi)\right|^{2}}{1-|\Gamma|^{2}} \frac{1}{\left|\alpha^{\prime}\right|^{2}},
$$

where $\Gamma$ is the reflection coefficient of the antenna and $\vec{K}_{s m n}(\theta, \phi)$ is the far-field pattern function [24]. Assuming the antenna to be $100 \%$ efficiency, the integration of the gain on all solid angles must be $4 \pi$. Therefore $\alpha^{\prime}$ is determined as

$$
\left|\alpha^{\prime}\right|^{2}=\frac{\int\left|\sum_{s m n} \alpha T_{s m n} \vec{K}_{s m n}(\theta, \phi)\right|^{2} d \Omega}{4 \pi\left(1-|\Gamma|^{2}\right)} .
$$




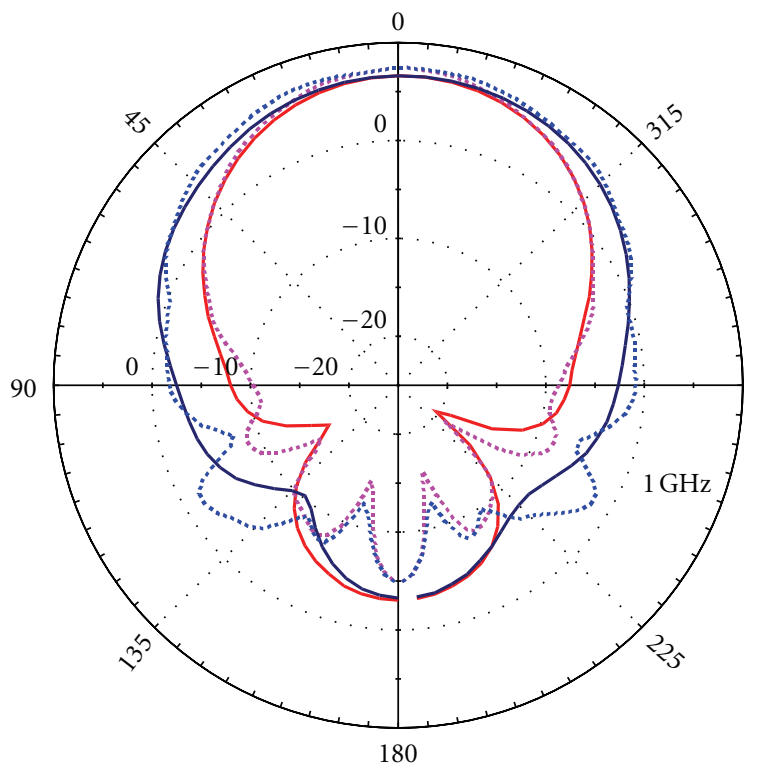

(a) $1 \mathrm{GHz}$

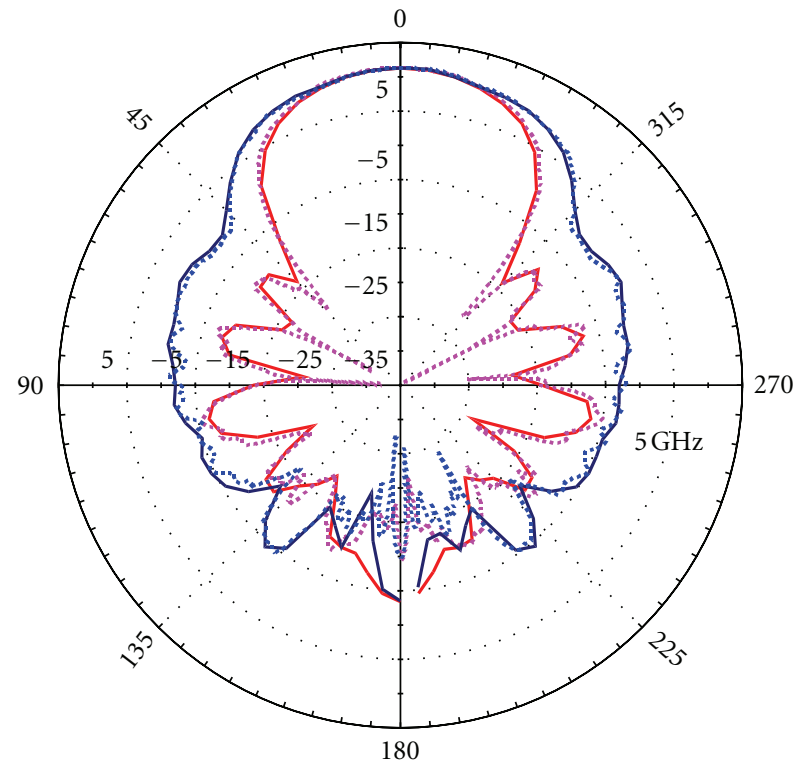

(b) $5 \mathrm{GHz}$

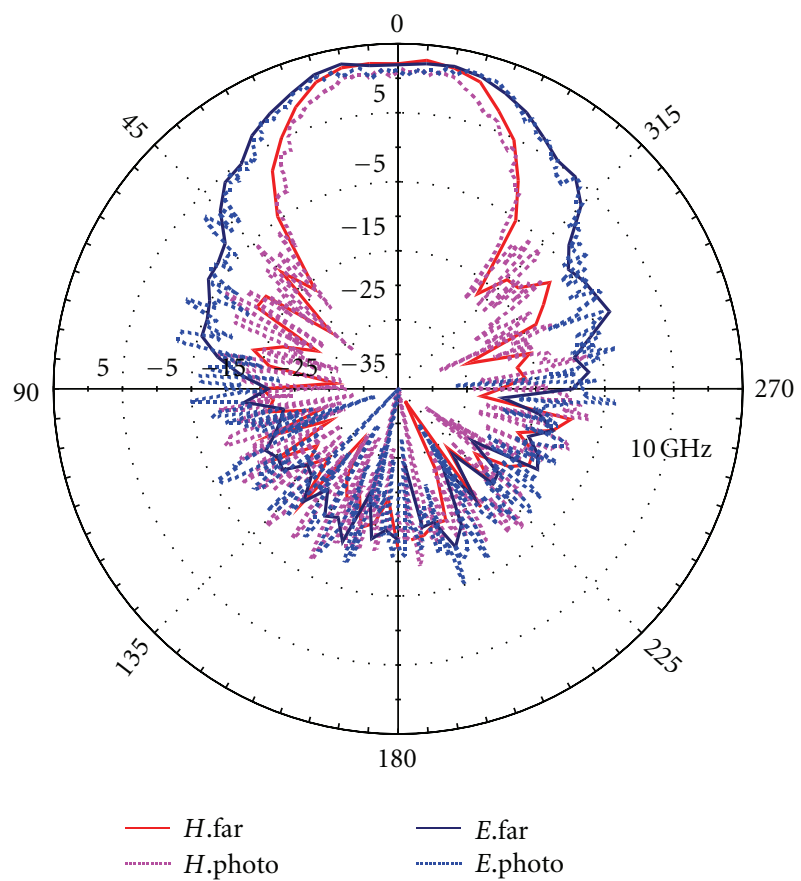

(c) $10 \mathrm{GHz}$

FIGURE 18: Absolute gain patterns in the $\mathrm{E}$ and $\mathrm{H}$ planes, obtained by the far-field method (far), and the proposed method using the photonic sensor (photo).

Inserting $\left|\alpha^{\prime}\right|^{2}$ in the denominator of (6), the absolute gain pattern is finally obtained.

To demonstrate the validity of the proposed method, a double-ridged waveguide horn antenna (Model 3115 manufactured by EMCO) was used because the antenna efficiency can be assumed to be $100 \%$. The absolute boresight gain obtained by the proposed method is shown in Figure 17. For comparison, the absolute gain by the far-field three antenna method is also shown. The both absolute gains agree with each other within $1 \mathrm{~dB}$ below $10 \mathrm{GHz}$. The error of the method comes from the pattern error: the error below $2 \mathrm{GHz}$ is due to the scattering around equipment as in Figure 18(a); the error above $9 \mathrm{GHz}$ is due to the noise as in Figure 18(c). If those factors can be reduced, the error of the method will be reduced to about $0.3 \mathrm{~dB}$ or less in our experiences.

In Figure 18, the patterns of the absolute gains by both methods in the $\mathrm{E}$ plane and the $\mathrm{H}$ plane are shown at $1 \mathrm{GHz}$, $5 \mathrm{GHz}$, and $10 \mathrm{GHz}$. They agree well with each other. 


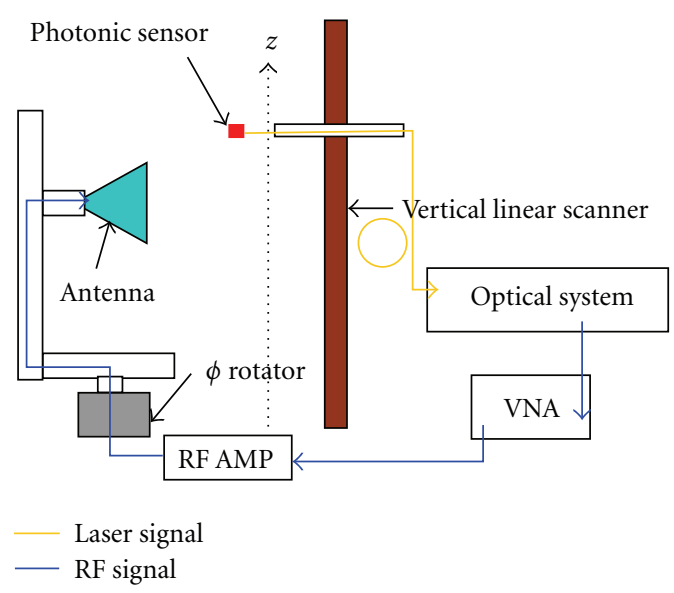

FIgURE 19: Measurement setup for cylindrical near-field scanning.

From the results, the photonic sensor can be considered to be an infinitesimal receiving antenna below $10 \mathrm{GHz}$ in spherical near-field measurements as well as planar near-field measurements.

3.3. Cylindrical Near-Field Measurements. Figure 19 shows a cylindrical near-field measurement system using the photonic sensor. Because the polarizations of the sensor are set to be parallel to $E_{\phi}$ and $E_{z}$, the measured $S_{21} s$ are proportional to each component and expressed as [25]

$$
S_{21 z}(R, \phi, z)=\alpha_{c} \sum_{n=-\infty}^{\infty} e^{j n \phi} \int_{-\infty}^{+\infty} b_{n}(h) \frac{\Lambda^{2}}{k} H_{n}^{(2)}(\Lambda R) e^{-j h z} d h,
$$

$$
\begin{aligned}
S_{21 \phi}(R, \phi, z) \\
=\alpha_{c} \sum_{n=-\infty}^{\infty} e^{j n \phi} \\
\quad \times \int_{-\infty}^{+\infty}\left[b_{n}(h) \frac{n h}{k R} H_{n}^{(2)}(\Lambda R)-a_{n}(h) \frac{\partial H_{n}^{(2)}(\Lambda R)}{\partial R}\right] \\
\quad \times e^{-j h z} d h,
\end{aligned}
$$

where $\alpha_{c}$ is a constant, $(R, \phi, z)$ is the position of the sensor, $H_{n}^{(2)}$ is the Hankel function of the second kind, and $\Lambda=$ $\sqrt{k^{2}-h^{2}}$. The coefficients $a_{n}(h)$ and $b_{n}(h)$ are determined by the Fourier transform.

Since the normalized electric far-field is given as

$$
\begin{aligned}
& \mathbf{E}(\theta, \phi) \\
& \quad=-2 k \sin \theta \sum_{n=-\infty}^{\infty} j^{n} e^{j n \phi}\left[\hat{\boldsymbol{\varphi}} a_{n}(k \cos \theta)+\hat{\boldsymbol{\theta}} j b_{n}(k \cos \theta)\right],
\end{aligned}
$$

the absolute gain pattern is obtained by

$$
G(\theta, \phi)=\frac{16 \pi Z_{0} k^{2}}{Z_{\text {free }}\left(1-|\Gamma|^{2}\right)}|\mathbf{E}(\theta, \phi)|^{2}
$$

where $Z_{0}$ and $\Gamma$ are the characteristics impedance of the cable and the reflection coefficient of the antenna, respectively.

To verify the equations from (8) up to (11), a standard horn antenna (MI 12-1.7 manufactured by MI Technologies) at $1.7 \mathrm{GHz}$ was measured by a cylindrical scanner (Model 300 $\mathrm{V}-8 \mathrm{x} 8$ manufactured by NSI). The E plane of the antenna is along the $\phi$ axis and the $\mathrm{H}$ plane is parallel to the $z$ axis. Figure 20 shows the absolute gain patterns of the horn antenna obtained by the conventional WR430 OEW probe, FEKO, and the photonic sensor. Since the photonic sensor system (at present) cannot measure the absolute gain, the relative patterns whose maximum is set to the maximum of the patterns by the OEW probe are shown. The results by the three methods agree with each other. The differences between FEKO and the measurements probably come from the electromagnetic absorber around the horn antenna that was used only for the measurements.

Figure 21 shows the relative phase patterns of $E \phi$ obtained by FEKO, the photonic sensor, and the OEW probe. $E \phi$ is the principal component in the setting of the antenna. To calculate each phase, the origin of each coordinate system is set to the centre of the antenna aperture and each phase at $\left(\phi, k_{z}\right)=(0,0)$ is set to that of FEKO. Those by FEKO and the sensor agree well with each other. Slight difference in the OEW probe may be error in the characterization of the OEW probe or the setting of the antenna in the measurement.

The measurement conditions for the OEW probe are determined to satisfy the criteria [1] where the distance between the aperture of the antenna and that of the OEW probe is $50 \mathrm{~cm}, R=0.96 \mathrm{~m}, \phi=-157.5$ degrees To +157.5 degrees, $\Delta \phi=3.75$ degrees, $z=-1.188 \mathrm{~m}$ to $+1.188 \mathrm{~m}, \Delta z=$ $0.066 \mathrm{~m}$. The measurement by the photonic sensor was done on the conditions where the distance between the aperture and the sensor is $6.5 \mathrm{~cm}, R=0.5 \mathrm{~m}, \phi=-152$ degrees To +152 degrees, $\Delta \phi=4$ degrees, $z=-1.08 \mathrm{~m}$ to $+1.08 \mathrm{~m}, \Delta z=$ $0.036 \mathrm{~m}$. The model calculated by FEKO has the parameters where the aperture and the height of the horn is $367 \mathrm{~mm} \times$ $273 \mathrm{~mm} \times 265 \mathrm{~mm}$, the waveguide size and the length are $109.2 \mathrm{~mm} \times 54.6 \mathrm{~mm} \times 150 \mathrm{~mm}$, the meshing size is $10 \mathrm{~mm}$.

\section{Summary}

We have demonstrated the utilities of the photonic sensor applied to planar, spherical, and cylindrical near-field antenna measurements. The photonic sensor consists of the array antenna with $2.4 \mathrm{~mm}$ height and $2 \mathrm{~mm}$ width on $\mathrm{LiNbO}_{3}$ substrate and operates in the principle of the Mach-Zehnder interferometer. Due to the physical smallness, the photonic sensor can be considered to be an infinitesimal receiving antenna below $10 \mathrm{GHz}$. Therefore no probe compensation is required for any kinds of near-field measurements.

As for the sensitivity, the overall minimum sensitivity of the present photonic sensor system is $0.5 \mathrm{~V} / \mathrm{m}$ approximately. This means that the system can be used to measure an antenna whose gain is over $10 \mathrm{~dB}$, at about $50 \mathrm{~mm}$ apart from the aperture of the antenna, using a vector network analyzer in SNR over $30 \mathrm{~dB}$ with an RF amplifier of about $30 \mathrm{~dB}$ gain. 


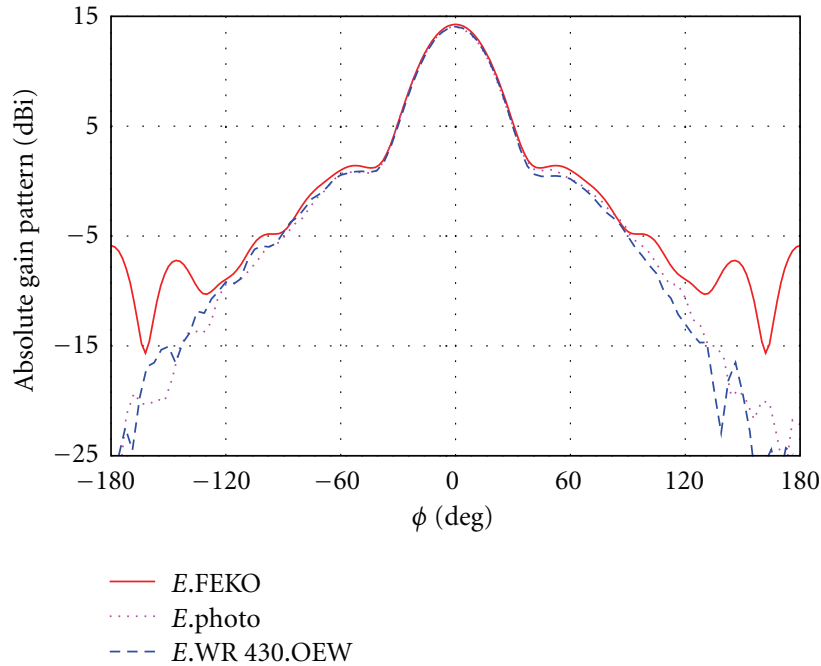

(a) E-plane

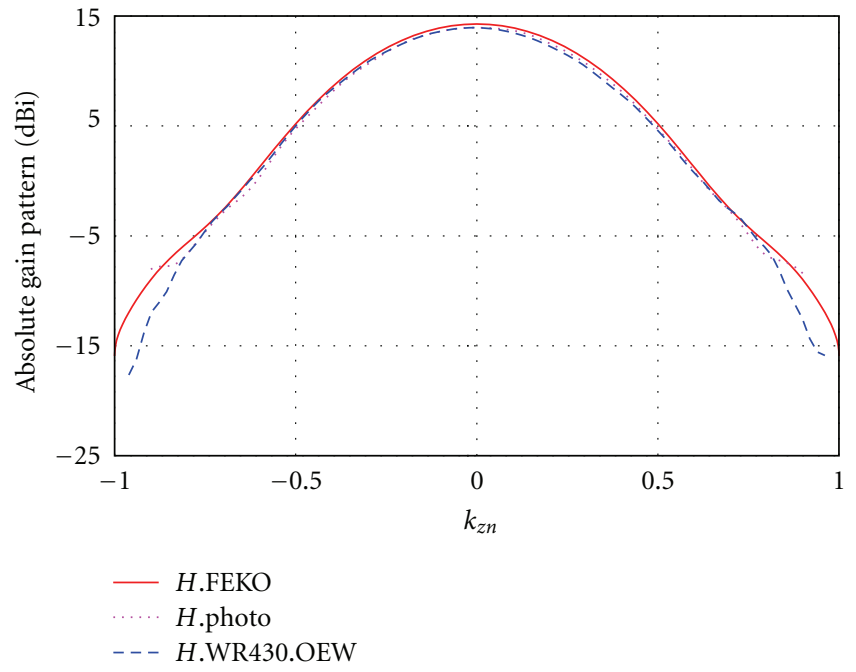

(b) H-plane

FIGURE 20: Absolute gain patterns of the standard horn antenna obtained by the OEW (WR430) probe, FEKO, and the relative pattern of the photonic sensor at $1.7 \mathrm{GHz}$. (a) E-plane. (b) H-plane.

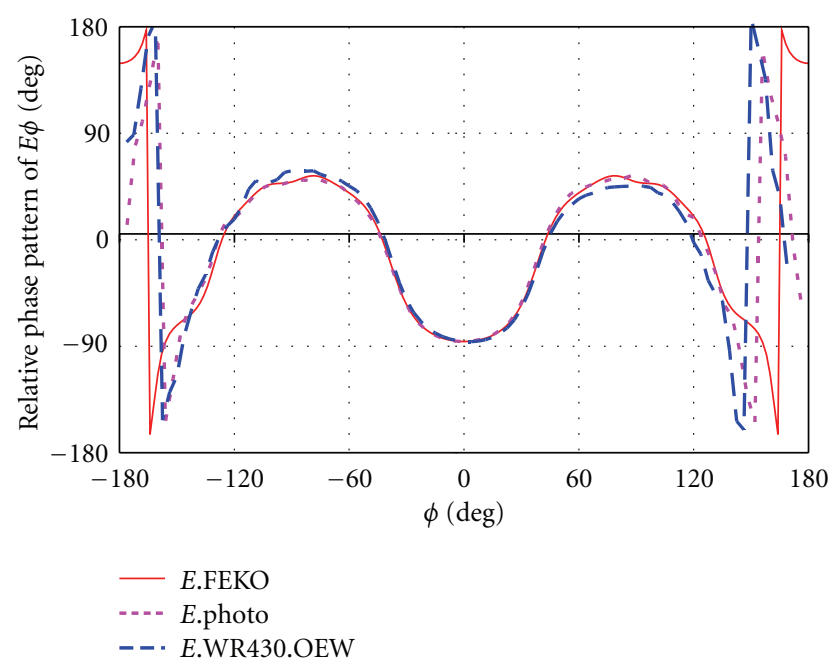

(a) E-plane

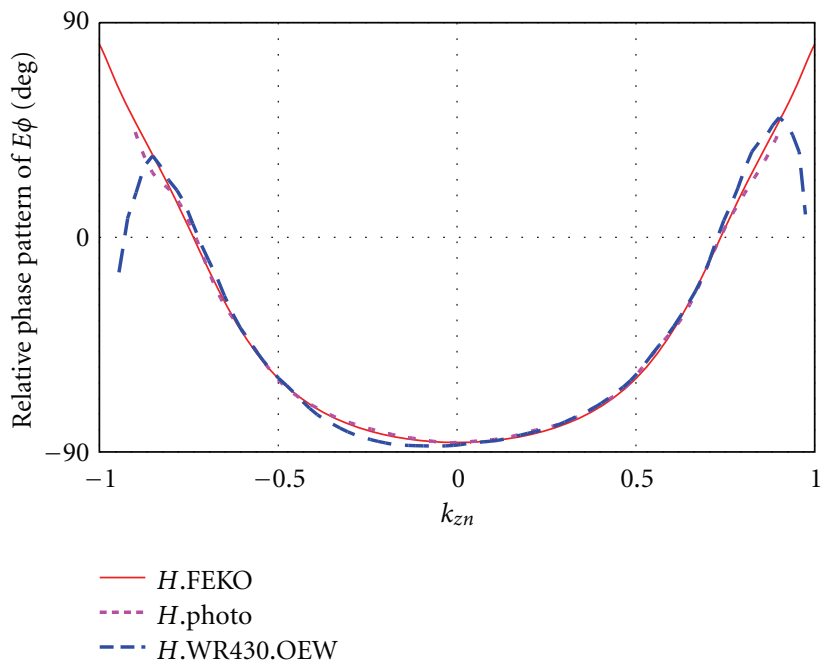

(b) H-plane

FIGURE 21: Relative phase patterns of $E \phi$ (principal component) of the standard horn antenna obtained by the OEW (WR430) probe, FEKO, and the photonic sensor at $1.7 \mathrm{GHz}$. (a) E-plane. (b) H-plane.

To summarise, the merits of the photonic sensor applied to near-field antenna measurements are

(1) no probe compensation is required below $10 \mathrm{GHz}$,

(2) broad band measurements from a few tens $\mathrm{MHz}$ to $10 \mathrm{GHz}$ are realized by the single probe,

(3) the sensor can be treated as the more ideal infinitesimal receiving antenna if the operating frequency becomes the lower,

(4) the truncation error can be reduced,

(5) evanescent waves in the immediate vicinity of the aperture can be detected,
(6) measurement system can be made in small and compact size.

We are continuously developing a new optical system that will have the overall minimum sensitivity of $1 \mathrm{mV} / \mathrm{m}$ and need no RF amplifier. The system combined with a VNA will realize antenna measurements calibrated by various kinds of calibration methods.

\section{References}

[1] A. D. Yaghjian, "An overview of near-field antenna measurements," IEEE Transactions on Antennas and Propagation, vol. 34, no. 1, pp. 30-45, 1986. 
[2] CISPR 16-1-4, "Specification for radio disturbance and immunity measuring apparatus and methods-part 1-4: radio disturbance and immunity measuring apparatus-ancillary equipment-radiated disturbances," International Electrotechnical Commission IEC, Edition 1.1, 2004-2005.

[3] S. Battermann and H. Garbe, "Influence of the receiving antenna pattern on the Site-VSWR validation procedure above $1 \mathrm{GHz}$," in Proceedings of the IEEE International Symposium on EMC, pp. 1-5, August 2008.

[4] M. Hirose, T. Ishizone, and K. Komiyama, "Antenna pattern measurements using photonic sensor for planar near-field measurement at X band," IEICE Transactions on Communications, vol. 87, no. 3, pp. 727-734, 2004.

[5] M. Hirose, S. Kurokawa, and K. Komiyama, "Compact spherical near-filed measurement system for UWB antennas," in Proceedings of the IEEE International Symposium on Antennas and Propagation (ISAP '05), vol. 4, pp. 692-695, July 2005.

[6] A. Capozzoli, C. Curcio, G. D'Elia et al., "Photonic probes and advanced (also phaseless) near-field far-field techniques," IEEE Antennas and Propagation Magazine, vol. 52, no. 5, pp. 232$241,2010$.

[7] M. Schwerdt, J. Berger, B. Schüppert, and K. Petermann, "Integrated optical E-Field sensors with a balanced detection scheme," IEEE Transactions on Electromagnetic Compatibility, vol. 39, no. 4, pp. 386-390, 1997.

[8] K. Tajima, R. Kobayashi, N. Kuwabara, and M. Tokuda, "Improving design method for sensitivity and frequency response of e-field sensor using a mach-zehnder interferometer," IEICE Transactions on Electronics, vol. 83, no. 3, pp. 347-354, 2000.

[9] T. Ichikawa, "Fabrication and characterization of a retroreflective type of practical $\mathrm{LiNbO}_{3}$ voltage sensor operating in the range of $6 \mathrm{~Hz}$ to $2 \mathrm{GHz}$," IEICE Transactions on Electronics, vol. 83, no. 3, pp. 355-359, 2000.

[10] A. I. Sasaki and T. Nagatsuma, "Electric-field scanning system using electro-optic sensor," IEICE Transactions on Electronics, vol. 86, no. 7, pp. 1345-1351, 2003.

[11] E. Suzuki, S. Arakawa, H. Ota, K. I. Arai, and R. Sato, "Optical magnetic field probe with a loop antenna element doubly loaded with $\mathrm{LiNbO}_{3}$ crystals," IEICE Transactions on Electronics, vol. 87, no. 11, pp. 1989-1996, 2004.

[12] H. Togo, N. Kukutsu, N. Shimizu, and T. Nagatsuma, "Sensitivity-stabilized fiber-mounted electrooptic probe for electric field mapping," Journal of Lightwave Technology, vol. 26, no. 15 , pp. 2700-2705, 2008.

[13] D.-J. Lee, N.-W. Kang, J.-H. Choi, J. Kim, and J. F. Whitaker, "Recent advances in the design of electro-optic sensors for minimally destructive microwave field probing," Sensors, vol. 11, no. 1, pp. 806-824, 2011.

[14] D. M. Kern, Plane-Wave Scattering-Matrix Theory of Antennas and Antenna-Antenna Interactions, chapter 2, National Bureau of Standards Monographs, 1981.

[15] R. E. Collin, Antennas and Radiowave Propagation, chapter 5, McGraw-Hill, 1985.

[16] http://www.feko.info/.

[17] A. C. Newell and C. F. Stubenrauch, "Effect of random errors in planar near-field measurement," IEEE Transactions on Antennas and Propagation, vol. 36, no. 6, pp. 769-773, 1988.

[18] J. B. Hoffman and K. R. Grimm, "Far-field uncertainty due to random near-field measurement error," IEEE Transactions on Antennas and Propagation, vol. 36, no. 6, pp. 774-780, 1988.

[19] J. Romeu, L. Jofre, and A. Cardama, "Far-field errors due to random noise in cylindrical near-field measurements," IEEE Transactions on Antennas and Propagation, vol. 40, no. 1, pp. 79-84, 1992.
[20] M. Hirose, K. Komiyama, J. Ichijo, and S. Torihara, "Pattern measurement of X-band standard gain horn antenna using photonic sensor and planar near field scanning technique," in Proceedings of the Antenna Measurement Techniques Association Symposium (AMTA '02), pp. 284-288, 2002.

[21] M. Hirose, T. Ishizone, J. Ichijoh, S. Torihata, and K. Komiyama, "Planar near-field measurements of microstrip array antenna using photonic sensor at X band," in Proceedings of the IEEE International Symposium on Antennas and Propagation (ISAP '03), vol. 4, pp. 67-70, 2003.

[22] M. Hirose, S. Kurokawa, and K. Komiyama, "A probe calibration method to measure absolute gain by spherical nearfield measurement system using photonic sensor," in Proceedings of the IEEE International Symposium on Antennas and Propagation (ISAP '05), vol. 1, pp. 277-280, Seoul, Korea, October 2005.

[23] M. Kanda and S. F. Kawalko, "Near-zone gain of $500 \mathrm{MHz}$ to 2.6 GHz rectangular standard pyramidal horns," IEEE Transactions on Electromagnetic Compatibility, vol. 41, no. 2, pp. 8592, 1999.

[24] J. E. Hansen, Ed., Spherical Near-Field Antenna Measurements, vol. 26 of IEE Electromagnetic Waves Series, Peter Peregrinus, London, UK, 1998.

[25] O. M. Bucci and C. Gennarelli, "Use of sampling expansions in near-field-far-field transformation: the cylindrical case," IEEE Transactions on Antennas and Propagation, vol. 36, no. 6, pp. 830-835, 1988. 

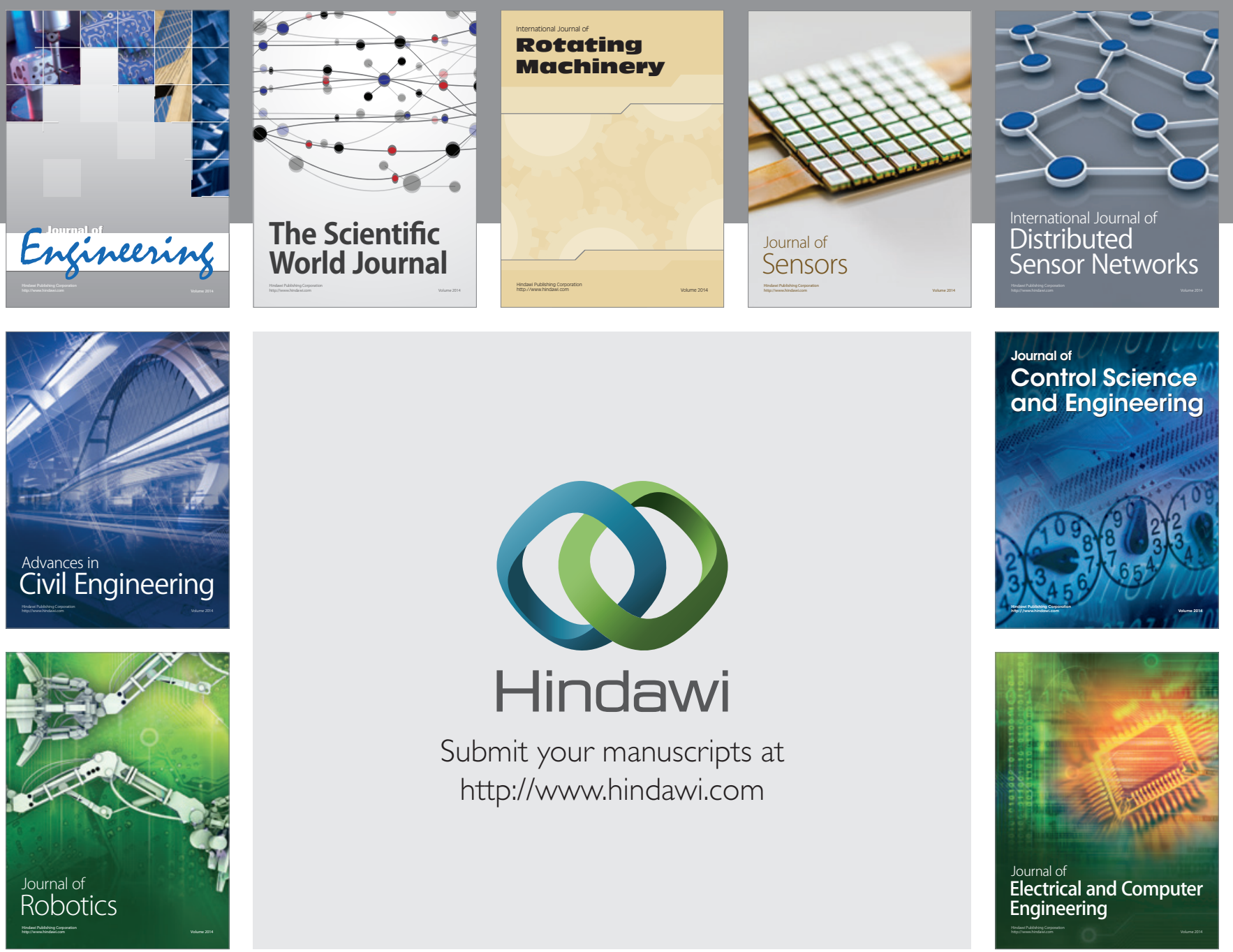

Submit your manuscripts at

http://www.hindawi.com
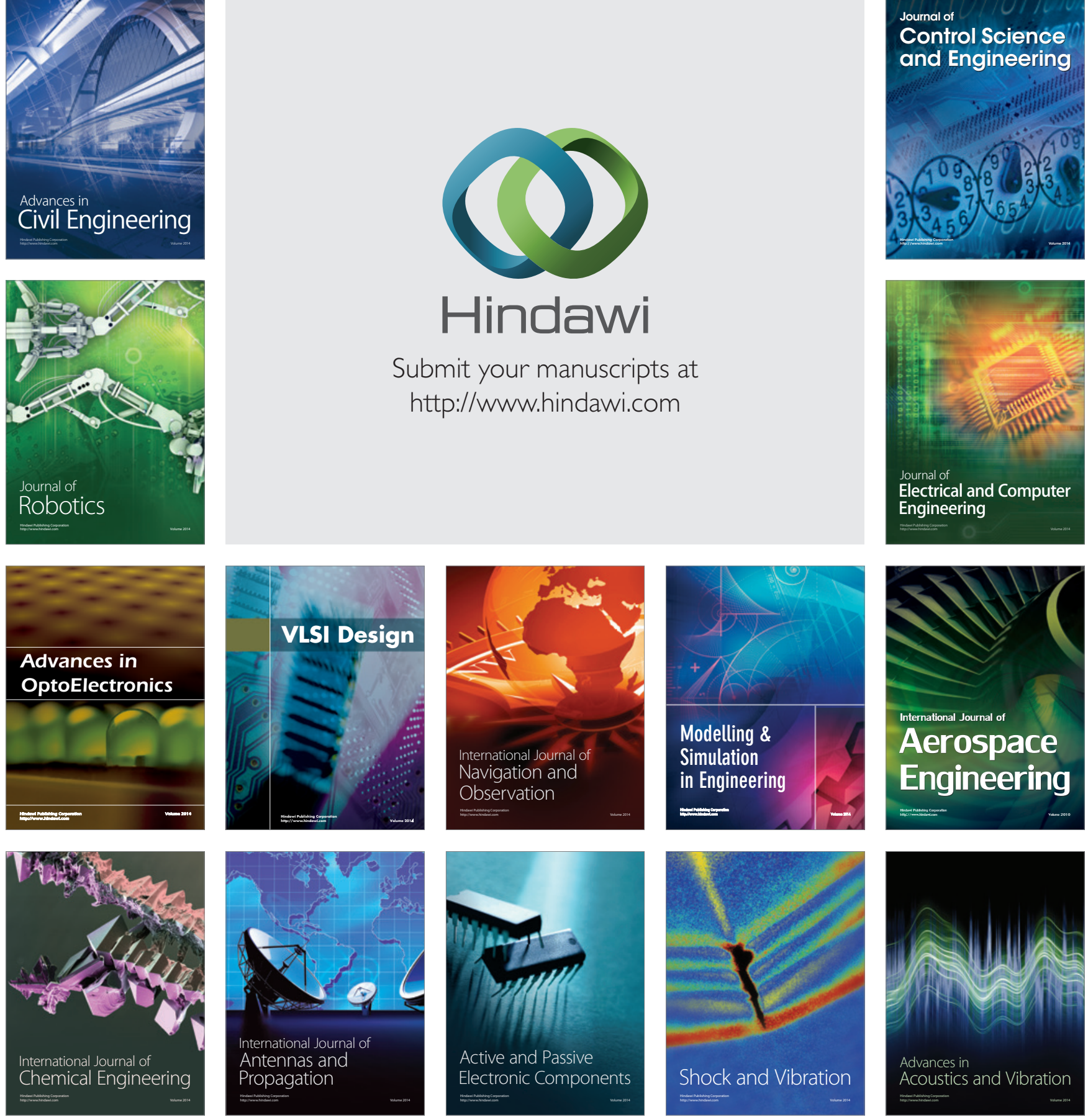